\title{
Milk exosomes and miRNA cross the placenta and promote embryo survival in mice
}

\author{
Mahrou Sadri ${ }^{1}$, Jiang Shu², Stephen D Kachman³, Juan Cui and Janos Zempleni®1 \\ ${ }^{1}$ Department of Nutrition and Health Sciences, University of Nebraska-Lincoln, Lincoln, Nebraska, USA, \\ ${ }^{2}$ Department of Computer Science and Engineering, University of Nebraska-Lincoln, Lincoln, Nebraska, USA and \\ ${ }^{3}$ Department of Statistics, University of Nebraska, Lincoln, Nebraska, USA
}

Correspondence should be addressed to J Zempleni; Email: jzempleni2@unl.edu

\begin{abstract}
Exosomes facilitate cell-to-cell communication by transferring regulatory molecules such as miRNA from donor to recipient cells, for example, miR-21-5p and miR-30d promote placentation. Exosomes and their miRNA cargos are not exclusively obtained from endogenous synthesis but may also be absorbed from dietary sources, such as milk. This study assessed the effects of milk exosomes and miRNA cargos on embryo development and fertility in C57BL/6 mice. Fluorophore-labeled milk exosomes, miR-21-5p and miR-30d accumulated in murine placenta and embryos following oral gavage. Seventeen mRNAs, miR-21-5p and miR-30d were differentially expressed in placentas of pregnant mice fed a milk exosome and RNA-depleted (ERD) diet or a milk exosome and RNA-sufficient (ERS) diet. Eight of these mRNAs encode proteins implicated in the synthesis of extracellular matrix components, cell adhesion and migration. Changes in mRNA expression were associated with corresponding changes in protein expression, for example, collagen type I. The size of litters born to dams fed ERD was 25-50\% smaller than those born to ERS controls. This study implicates dietary exosomes and miRNA in placenta development and embryo survival.

Reproduction (2020) $160501-509$
\end{abstract}

\section{Introduction}

Exosomes are extracellular vesicles, size 30-150 nm, that facilitate cell-to-cell communication by transferring regulatory cargos such as mRNAs, miRNA, proteins, and lipids from donor cells to adjacent or distant recipient cells (Yanez-Mo et al. 2015, Gurunathan et al. 2019). Among exosome cargos, miRNA are of particular interest because they regulate more than $60 \%$ of human genes by binding to complementary sequences in the $3^{\prime}$-UTRs in mRNAs, and loss of miRNA maturation in Dicer knockout mice is embryonic lethal (Bernstein et al. 2003, Friedman et al. 2009). Extracellular vesicles, including exosomes, have been implicated in gamete maturation, fertilization and embryo implantation, and their miRNA cargos regulate oocyte developmental competence (Sohel et al. 2013, Machtinger et al. 2016). For example, miR-21-5p plays a key role in the implantation of fertilized eggs and early embryonic development in mice, and exosome-associated and free miR-30d is taken up by murine embryos leading to an increase in adhesion to the uterine epithelium (Vilella et al. 2015, Lv et al. 2018).

Classically, exosomes and their miRNA cargos were considered endogenous regulators of gene expression and metabolism. In previous studies, we demonstrated that exosomes and their miRNA cargos may also be absorbed from milk, including the absorption of bovine exosomes and miRNA across species boundaries in humans and mice (Baier et al. 2014, Manca et al. 2018). Subsequently, we and at least five independent laboratories confirmed and expanded this discovery in follow-up studies (Zhang et al. 2012, Shu et al. 2015, Wolf et al. 2015, Chen et al. 2016, Munagala et al. 2016, Golan-Gerstl et al. 2017, Liao et al. 2017, Manca et al. 2018). These studies included the development of a technology that used bovine milk exosomes (BMEs) transfected with fluorophore (IRDye)-labeled miRNA to assess the tissue distribution of dietary miRNA (Manca et al. 2018). Distinct miRNA displayed unique distribution patterns but liver, brain, intestinal mucosa and kidneys were the primary sites of accumulation in non-pregnant mice. In the spirit of transparency, it is worthwhile mentioning that some studies failed to detect the absorption of miRNA from the milk of reported low bioavailability; these studies and their limitations have recently been reviewed (Zempleni et al. 2019). Encapsulation in BMEs protects miRNA against degradation by low $\mathrm{pH}$ and enzymes in the gastrointestinal tract and the harsh conditions applied in the processing of milk in dairy plants (Izumi et al. 2012, Howard et al. 2015, Benmoussa et al. 2016). Encapsulation also confers a pathway for miRNA 
absorption through endocytosis of the BME shell (Wolf et al. 2015, Kusuma Jati et al. 2016).

BMEs harbor more than 400 mature miRNA, including miR-21-5p and miR-30d (Kosaka et al. 2010, Sun et al. 2015). miRNA are highly conserved among mammals and nucleotide sequences are identical in most of the human, murine and bovine miRNA (Kozomara \& Griffiths-Jones 2014). Sequence conservation is consistent with reports that bovine miRNA encapsulated in BMEs alter gene expression in human peripheral blood mononuclear cells ex vivo, human embryonic kidney 293 cells, and murine liver and skeletal muscle (Baier et al. 2014, Aguilar-Lozano et al. 2018, Leiferman et al. 2018). Endogenous synthesis of miRNA cannot compensate for the depletion of BMEs and their miRNA cargos. For example, when mice were fed an exosome and RNA-depleted (ERD) diet, plasma concentrations of miRNA decreased by more than $60 \%$ compared with controls fed an exosome and RNA-sufficient (ERS) diet (Baier et al. 2014, Aguilar-Lozano et al. 2018). Phenotypes of milk exosome and RNA cargo depletion include an increase of purine metabolites in human and murine tissues and body fluids, moderate loss of muscle grip strength in mice and a decrease in villus height and crypt depth in the porcine intestinal mucosa (Chen et al. 2016, Aguilar-Lozano et al. 2018, Leiferman et al. 2018).

Here we tested the hypothesis that BMEs and their miRNA cargos promote fecundity in mice. The objectives of this study were to (1) assess the accumulation of BMEs and their miRNA cargos in murine embryos and placenta following oral administration, (2) assess effects of BMEs and miRNA on the expression of genes and pathways implicated in placenta development during murine gestation, and (3) determine whether litter size and postnatal survival are decreased in mice fed an ERD diet compared with mice fed an ERS diet.

\section{Materials and methods}

\section{Mouse husbandry}

C57BL/6 mice, aged 3 weeks, were purchased from Jackson Laboratories (stock No: 000364) and kept in a $12 \mathrm{~h}$ light:12 h darkness cycle at ambient temperature. Mice used in breeding studies were fed exosome and RNA-defined diets. The ERS and ERD diets used in this study are based on the AIN-93G formulation modified to provide a number of exosomes and cargos equivalent to $0.5 \mathrm{~L}$ bovine milk consumed by a human adult (Reeves 1993, Baier et al. 2014). The milk used to prepare the ERD diet was ultrasonicated for $1.5 \mathrm{~h}$ and incubated at $37^{\circ} \mathrm{C}$ for $1 \mathrm{~h}$ prior to pelleting the diet, whereas the milk used to prepare the ERS diet was not ultrasonicated (Leiferman et al. 2018). Ultrasonication caused a transient opening of the exosome membrane leading to a $20 \%$ loss of exosomes, a near-complete loss of miRNA cargos, a more than $60 \%$ loss of exosome transport by intestinal Caco- 2 cells and a more than $60 \%$ decrease in plasma miRNA concentrations in mice fed ERD compared with ERS in previous studies
(Baier et al. 2014, Leiferman et al. 2018). In studies of dietary effects on litter size, mice were mated at the age of 15 weeks in all possible permutations of males and females fed ERS and ERD diets; ERS males mated to ERS females served as controls. Mice fed AIN-93G diet were used as controls in select experiments. Males were separated from females after mating, judged by the presence of a vaginal plug. Live pups were not counted until day 7 postpartum to avoid disruption of maternal care, and survival to weaning was assessed at the age of 3 weeks. In studies of placental gene expression, ERS males were mated with ERS or ERD females at the age of 15 weeks. In studies of BMEs and miRNA accumulation in placentas and embryos, mice were started on 2016 Teklad global 16\% protein rodent diet (ENVIGO, Inc) at the age of 3 weeks and continued on the diet until mating at the age of 15 weeks. Time courses of experimental arms are shown in Fig. 1. All mice had free access to food and water. All studies were approved by the Institutional Animal Care Program at the University of Nebraska-Lincoln (protocol 1229).

\section{Labeling of BMEs and miRNA cargos}

BMEs were purified from fat-free milk from a local grocery store by using serial ultracentrifugation (Fiberlite F37L-8x100 rotor; Thermo Fisher Scientific, Inc) and authenticated by immunoblotting, size analysis (NanoSight NS300) and transmission electron microscopy as previously described (data not shown, Manca et al. 2018). BMEs from a representative experiment had a diameter of $144 \pm 6.0 \mathrm{~nm}$ (mean \pm s.D.), as per nanoparticle tracker analysis (NanoSight NS300, Malvern, Inc). Exosomes were labeled with 1,1-dioctadecyl-3,3,3,3tetramethylindotricarbocyanine iodide (DiR) by incubating $10^{12}$ BMEs with $5 \mu \mathrm{M}$ DiR in a volume of $1 \mathrm{~mL}$ at room temperature for $15 \mathrm{~min}$, followed by removing unbound dye and collecting labeled BMEs by ultracentrifugation. Lipophilic dyes such as DiR may transfer to lipophilic structures other than BMEs after administration to mice (Takov et al. 2017, Manca et al. 2018). This confounder can be avoided by transfecting BMEs with fluorophore-labeled miRNA (Manca et al. 2018). We loaded BMEs with synthetic IRDye-labeled miR-30d (5'-IRDye800-UGUAAACAUCCCCGACUGGAAGCU-3') or IRDye-labeled miR-21-5p (5'-IRDye800UAGCUUAUCAGACUGAUGUUGACU-3') (IDTDNA, Inc) by



Figure 1 Study schematic. 
incubation with $0.5 \mathrm{mM}$ calcium chloride and $40 \%$ ethanol for $15 \mathrm{~min}$ as previously described (Manca et al. 2018).

\section{Exosome and miRNA accumulation in placentas and embryo}

DiR-labeled BMEs and BMEs transfected with IRDye-labeled miRNA (controls) were administered to pregnant mice by oral gavage $\left(1 \times 10^{12} \mathrm{BMEs} / \mathrm{g}\right.$ body weight) on ED 16.5. Controls were prepared by administering BMEs not labeled with DiR or BMEs mixed with IRDye-labeled miRNA in the absence of transfection reagent. Mice were killed by carbon dioxide $\left(\mathrm{CO}_{2}\right)$ $24 \mathrm{~h}$ after oral gavage. Placentas and embryos were dissected and perfused with cold PBS to remove extracellular exosomes; litter size and sex of pups were not recorded. Fluorescence was assessed using LI-COR Odyssey ${ }^{\circledR}$ imaging system (LI-COR Biosciences, Lincoln, NE) in embryos and placentas (Manca et al. 2018).

\section{Quantitative reverse transcription (RT-qPCR)}

miRNA were isolated from placentas by using the miRNeasy Mini Kit (QIAGEN, Inc., cat. no. 217004) and reverse transcribed by using the miScript II RT kit (QIAGEN, Inc., cat. no. 218161) following the manufacturer's protocols. Synthetic cel-miR-39 was added after lysis reagent and used to normalize for extraction efficiency (Hromadnikova et al. 2017). Levels of cel-miR-39, miR-21-5p, miR-30d, miR-200c, and miR-148a were assessed by RT-qPCR as previously described (Leiferman et al. 2019). Data shown as fold-change values calculated following the delta delta Cq protocol.

\section{Gene expression in placenta}

Placentas and embryos were dissected from pregnant mice on ED 14.5 and their weights were recorded. The total RNA was extracted from ERS and ERD placentas and submitted to University of Nebraska Medical Center (UNMC) Sequencing Core facility for mRNA sequencing. Sequencing data analysis and functional enrichment analysis were conducted as previously described (Leiferman et al. 2018). Raw sequencing data have been deposited in NCBI BioProject (ID: PRJNA578367). Putative miRNA binding sites were identified by using miRTarBase (Huang et al. 2020).

\section{Western blot analysis}

Proteins (50 mg) were extracted from placentas by using RIPA buffer including proteinase inhibitor (100-fold final dilution, Thermo Fisher Scientific, Inc., cat. no. 89900). Protein concentrations were measured using the Pierce ${ }^{\mathrm{TM}}$ BCA Protein Assay Kit (Thermo Fisher Scientific, Inc.). After separation by SDS-PAGE, proteins were electroblotted to a nitrocellulose membrane. Membranes were blocked with 5\% BSA for $2 \mathrm{~h}$ at room temperature. COL1A1 and COL1A2 were probed using anti-COL1A1 (Santa Cruz Biotechnology, sc-59772; diluted 500-fold with 5\% BSA in PBS) and anti-COL1A2 (Santa Cruz Biotechnology sc-393573; diluted 500-fold with $5 \%$ BSA in PBS), respectively, at $4{ }^{\circ} \mathrm{C}$ overnight. $\beta$-Actin was used as housekeeping control (Cell Signaling, cat. no. 4967; diluted $1000-$ fold with $5 \%$ BSA in PBS). Membranes were washed three times with PBS $(0.1 \%$ Tween 20$)$ for $10 \mathrm{~min}$ and incubated with fluorophore-conjugated goat anti-mouse IgG (LI-COR, cat. no. 926-32210; diluted 2000-fold with 5\% BSA in PBS) at room temperature with gentle shaking for $1.5 \mathrm{~h}$. Membranes were washed three times and fluorescence was assessed by using a LI-COR Odyssey imager.

\section{Statistical analysis}

Differences in litter sizes and survival to weaning were assessed using Kruskal-Wallis test followed by the Conover post hoc test. Differences in fetal weight, placental weight and fetal-to-placental weight ratio among ERD and ERS and AIN-93G females were assessed using a one-way ANOVA with subsampling with a mouse as the experimental unit. Differences in fluorescence intensities in exosome, miRNA distribution studies, and Western blot analysis were assessed using either an unpaired $t$-test or a Welch's corrected $t$-test if an $F$ test for heterogeneity of variances was significant. Statistical analyses were performed using the GLIMMIX procedure in SAS 9.4 (SAS Institute Inc. 2013), R 3.5.0 (R Core Team 2018) and Prism 6 (GraphPad). Values are mean \pm S.E.M. or mean \pm S.D. $P<0.05$ was considered statistically significant.

\section{Results}

\section{Accumulation of BMEs and miRNA in murine placenta and embryo}

Pregnant $\mathrm{C} 57 \mathrm{BL} / 6$ mice absorb BMEs, and both BMEs and their miRNA cargos accumulate in placenta and embryos. When BMEs were labeled with the lipophilic fluorophore, DiR, and administered to pregnant mice by oral gavage on embryonic day (ED) 16.5 (day $0=$ mating), BMEs accumulated in placenta and embryo compared with controls treated with unlabeled BMEs on ED 17.5 (Fig. 2A). DiR has the disadvantage of transferring from exosomes to lipoproteins and proteins (Takov et al. 2017). Therefore, we also tracked the distribution of BMEs and their miRNA cargos by using BMEs transfected with fluorophore (IRDye)-labeled, synthetic miR-30d and miR-21-5p. In previous studies, we demonstrated that IRDye-labeled miRNA are stable and have distribution patterns comparable to those observed with other labeling technologies including exosomes endogenously labeled with fluorescent proteins (Manca et al. 2018). When BMEs were transfected with IRDyelabeled miR-30d and miR-21-5p and administered to pregnant mice by oral gavage on ED 16.5, BMEs accumulated in placenta and embryo compared with controls treated with non-transfected BMEs $6 \mathrm{~h}$ after gavage (Fig. 2B and C). Densitometry analysis suggested that the differences between DiR-labeled BME and unlabeled BME and between miRNA-transfected BME and non-transfected BME were statistically significant (Supplementary Table 1, see section on 

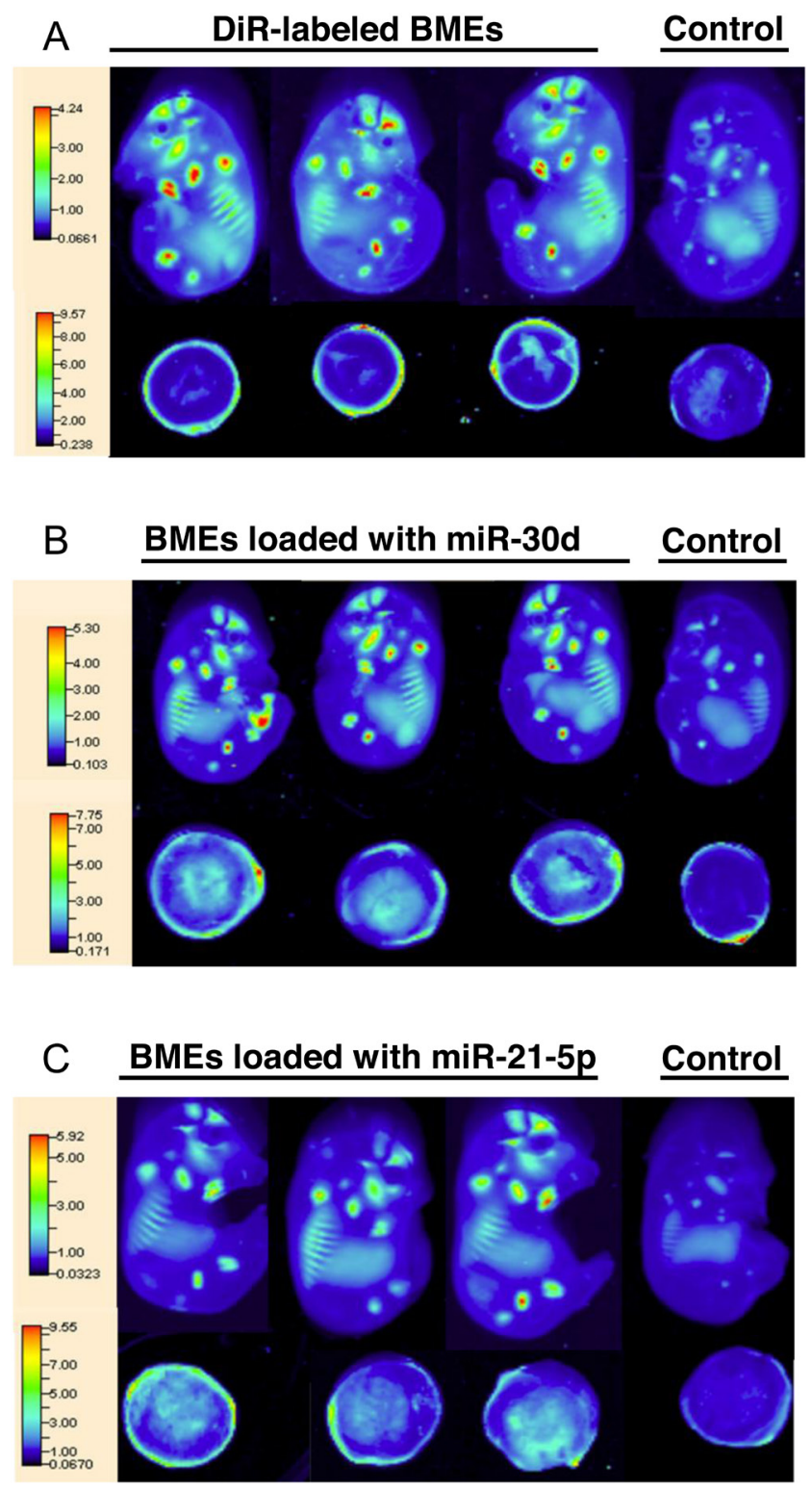

Figure 2 BMEs and miRNA accumulate in placenta and embryo. (A) DiR-labeled BMEs and unlabeled BMEs (control) in embryos (top row) and placentas (bottom row) on embryonic day (ED) 16.5 (24 h after oral BMEs administration to dams). (B) Fluorophore (IRDye)labeled miR-30d, transfected into BMEs, and non-transfected BMEs (control) in embryos (top row) and placentas (bottom row) in embryos $6 \mathrm{~h}$ after oral BMEs administration on ED 16.5. (C) Fluorophore (IRDye)-labeled miR-21-5p, transfected into BMEs, and nontransfected BMEs (control) in embryos (top row) and placentas (bottom row) in embryos $6 \mathrm{~h}$ after oral BMEs administration on ED 16.5. BMEs, bovine milk exosomes. ${ }^{*} P<0.05,{ }^{* *} P<0.01$, *** $P<0.001, n=3$ dams (from each dam, three embryos and three placentas were collected and analyzed). Representative examples from nine biological repeats from each treatment group are shown.

supplementary materials given at the end of this article). A punctuate pattern of BME and miRNA distribution was apparent in embryos, and fluorescence apparently localized to hard tissues.

\section{Effect of dietary BMEs and miRNA on miRNA concentrations in the placenta}

The concentration of miRNA in placentas depended on the dietary intake of BMEs and miRNA for the majority of the five amplicons tested. The miRNA were chosen because they are among the ten most abundant miRNA in BME (Cai et al. 2018). Levels of four miRNA, including miR-30d and miR-21-5p, were $50-80 \%$ lower in placentas from dams fed an exosome and RNAdepleted (ERD) diet compared to placentas from dams fed exosome and RNA-sufficient (ERS) diet (Fig. 3). The only exception was miR-320, for which the difference between groups ERD and ERS was not statistically significant. For all miRNA tested, concentrations were well within the detection limit, which was defined as a cycle threshold (Ct) value of 29.5 under the experimental conditions (Supplementary Table 2) (Wang et al. 2018).

\section{Effects of BMEs and RNA cargos on mRNA expression, weight and total protein in the murine placenta}

Seventeen mRNAs were differentially expressed in placentas from C57BL/6 mice fed ERD diet or ERS diet (Fig. 4A). Female mice were started on ERD or ERS diets at the age of 3 weeks and continued on these diets for 12 weeks when they were mated with male breeders (age of 15 weeks, fed ERS diet). The pregnant mice were continued on their diets and placentas were collected on ED 14.5 for mRNA expression by RNA-sequencing analysis. Gene ontology (GO) (cellular component) and Kyoto Encyclopedia of Genes and Genomes (KEGG) pathway analyses suggested that differentially expressed genes clustered in extracellular matrix components and cell adhesion pathways. These mRNAs were more abundant in ERS placentas than in ERD placentas (Fig. 4B). Collagen type I (COL1) is one of the main fibril collagens in placenta with a peak mRNA expression at about ED 14 (Arai and Nishiyama 2007). Effects of



Figure 3 Consumption of the ERD diet caused a depletion of miRNA in the placenta. miRNA in the placenta were analyzed by RT-qPCR. ERD, milk exosome and RNA-depleted diet; ERS, milk exosome and RNA-sufficient diet. ${ }^{*} P<0.05,{ }^{*} P<<0.01, n=3$ biological repeats per group. 
A
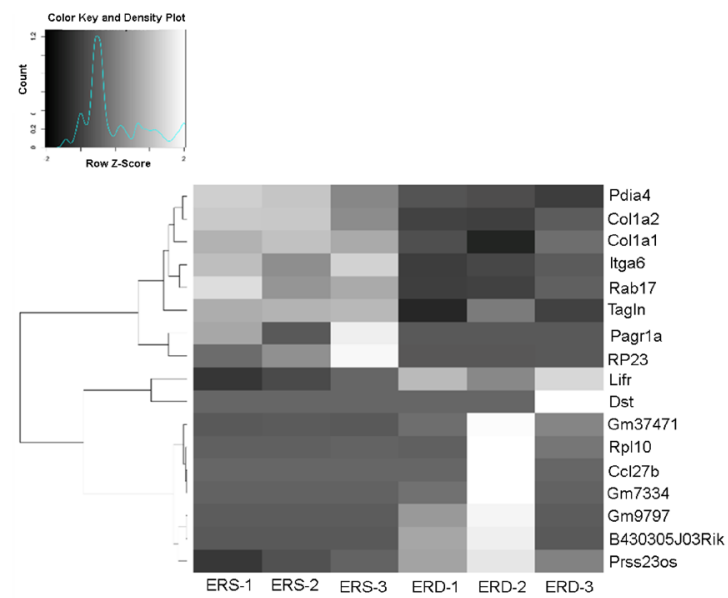

B
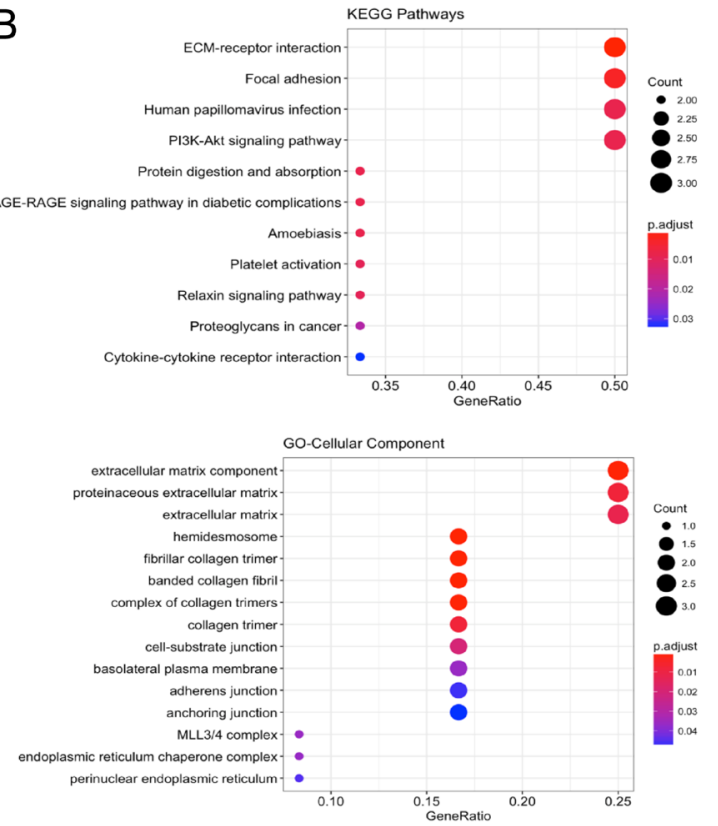

Figure 4 Genes were differentially expressed in placentas from mice fed ERD or ERS diets. (A) Differentially expressed mRNAs in ERD and ERS placentas on ED 14.5 ( $n=3$ biological repeats per group). Black and white colors reflected an increase and decrease in mRNA expression, respectively. GO enrichment analysis (B) and KEGG pathways analysis of differentially expressed mRNAs from ERD and ERS placentas. Dots represent the relationship of differentially expressed genes with cellular components. ECM, extracellular matrix; GO, Gene ontology; KEGG, Kyoto Encyclopedia of Genes and Genomes pathways; MLL3/4, mixed-lineage leukemia 3/4.

ERD and ERS feeding on COL1 Alpha 1 (COL1A1) and COL1 Alpha 2 (COL1A2) expression were confirmed at the protein level (Fig. 5). The 17 mRNAs that were differentially expressed in ERS and ERD placentas have 35 putative miRNA binding sites, which are targeted by 32 distinct miRNA (not shown). Nine of these miRNA were identified in BMEs in a previous study, miR-7e, miR-7i, miR-19b, miR-27a-3p, miR-27b, miR-128, miR29a, miR-29b and miR-362-3p (Benmoussa et al. 2020).

ERD placentas weighed significantly less in ERD females compared to ERS and AIN-93G controls on ED 14.5 (Fig. 6). Diet had no effect on total protein content in placentas and equaled $5.9 \pm 0.82,5.6 \pm 1.6$ and $4.8 \pm 1.2 \mathrm{mg} / \mathrm{mL}$ (means \pm s.D.) in groups AIN-93G, ERS, and ERD, respectively $(P>0.05)$.

\section{Effect of dietary BMEs and RNAs on litter size and survival to weaning}

Both litter size and survival to weaning were reduced in breeders and pups fed ERD compared to ERS and AIN93G controls. Note that male and female breeders were mated in all possible permutations by diet and pups were continued on maternal diets until weaning at the age of 3 weeks. The average litter size in ERS/ERS (female/ male) breeding pairs was approximately twice the size compared to breeders in which at least one parent was fed with the ERD diet (Fig. 7). The survival of pups to weaning time ranged from 0 to $50 \%$ when at least one parent was fed with ERD (Fig. 8).
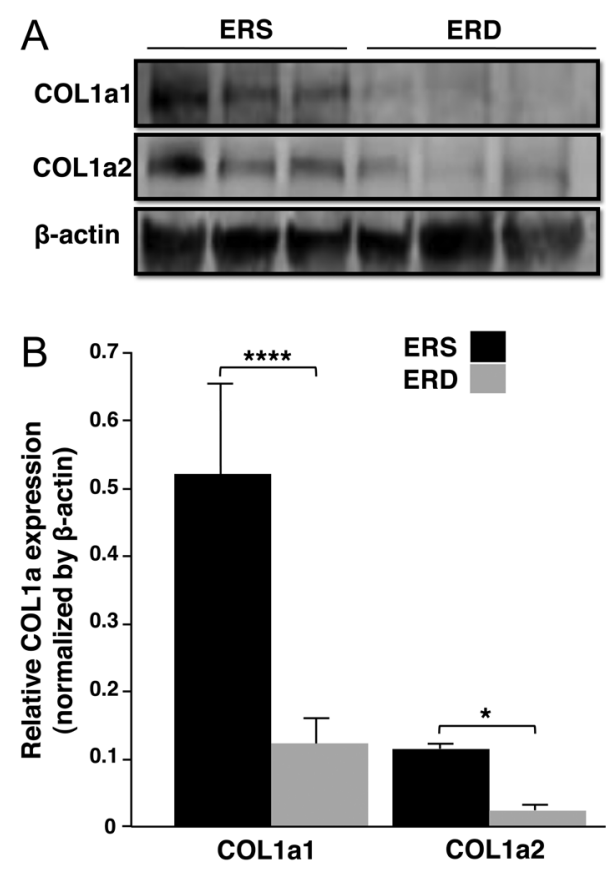

Figure 5 Expression of COL1A1 and COL1A2 decreased in placentas from mice fed the ERD diet. (A) Western blot analysis of COL1A1 and COL1A2 proteins in ERS and ERD placentas; $\beta$-actin was used as a loading control. (B) Gel densitometry analysis of COL1A1 and COL1A2 expression. COL1A1, collagen type I alpha I; COL1A2, collagen type I alpha $2 .{ }^{*} P<0.05,{ }^{* * * *} P<0.0001, n=3$ biological repeats per group. Values are mean \pm S.E.M. 


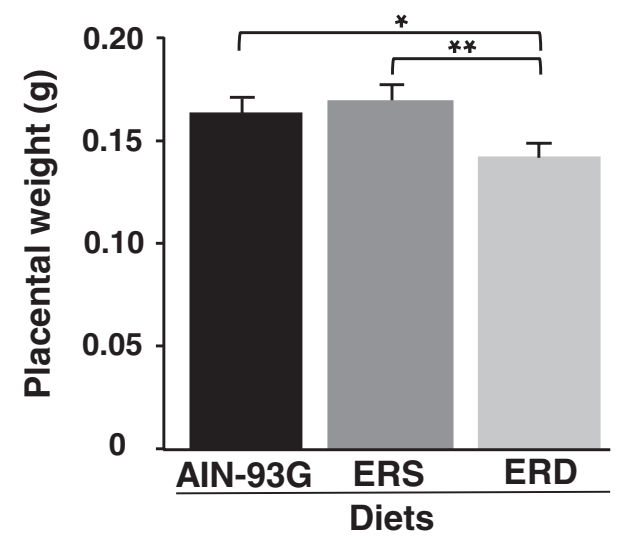

Figure 6 Placental weight was lower in dams fed the ERD diet compared to dams fed AIN-93G and ERS diets. Placental weights were measured on ED 14.5. ERD, milk exosome and RNA-depleted diet; ERS, milk exosome and RNA-sufficient diet. ${ }^{*} P<0.05$,

${ }^{* *} P<0.01$ vs control, $n=9$ pregnant dams per group. Values are mean \pm S.E.M.

\section{Discussion}

This paper is first to report an accumulation of orally administered BMEs and their miR-30d and miR-21-5p cargos in the murine placenta and embryos. Both miR30d and miR-21-5p are implicated in placentation and are among the top ten abundant miRNA in BMEs (Altmae et al. 2013, Vilella et al. 2015, Golan-Gerstl et al. 2017, Schanzenbach et al. 2017, Cai et al. 2018, Lv et al. 2018). In previous studies, we have shown that distinct miRNA delivered by BMEs have unique tissue distribution patterns, but the factors that regulate the partitioning of dietary miRNA among tissues are

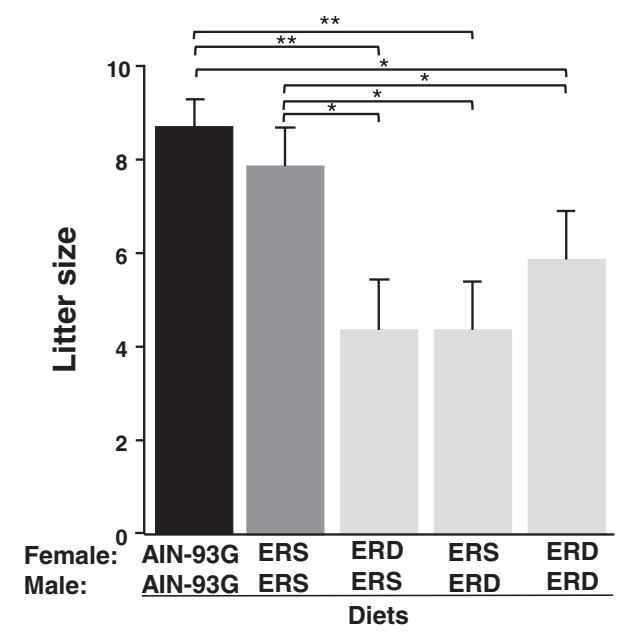

Figure 7 Litter sizes were smaller in C57BL/6 breeders fed the ERD diet compared to breeders fed the AIN-93G and ERS diet. ERD and ERS dams were bred in all possible permutations and live pups were counted on day 1 postpartum. ERD, milk exosome and RNA-depleted diet; ERS, milk exosome and RNA-sufficient diet. $* P<0.05$,

${ }^{* *} P<0.01$ vs control, $n=8$ breeder pairs per diet group. Values are mean \pm S.E.M.

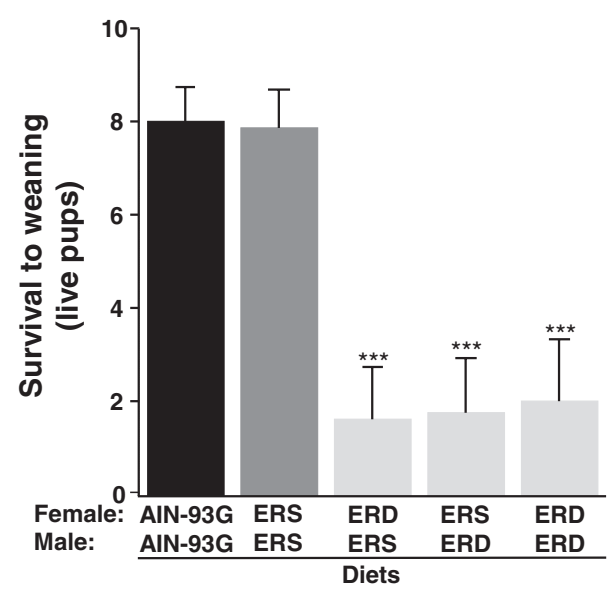

Figure 8 Survival of pups to weaning was smaller in mice fed the ERD diet compared to mice fed the AIN-93G and ERS diet. Live pups were counted 3 weeks postpartum. ERD, milk exosome and RNA-depleted diet; ERS, milk exosome and RNA-sufficient diet. $* * * P<0.001$ vs control, $n=8$ breeder pairs per diet group. Values are mean \pm S.E.M.

unknown (Manca et al. 2018). One possibility is that the abundance of complementary mRNAs regulates the accumulation of miRNA in tissues. In previous studies, we have extensively validated the technology used to assess the tissue distribution of miRNA in BMEs. For example, we used a combination of a fluorophore and quencher conjugation to synthetic miRNA to demonstrate that fluorophore-labeled miRNA are stable, and we used a variety of milk exosome and miRNA labeling techniques to validate distribution patterns (Manca et al. 2018). These studies suggest that the technology used here is robust and that we tracked miRNA rather than detached fluorophores. In contrast, studies that tracked exosomes by using lipophilic dyes need to be interpreted with caution, because those dyes may transfer from exosomes to proteins and lipoproteins (Takov et al. 2017). BMEs labeled with a lipophilic dye, DiR, failed to detect the accumulation of exogenous exosomes in the brain in previous studies, whereas alternative labeling technologies consistently showed accumulation of bovine and porcine milk exosomes and their miRNA cargos in the brain (Manca et al. 2018). While evidence has been accumulating that BMEs and their cargos in milk are bioavailable, some studies failed to detect the absorption of miRNA from the milk of reported low bioavailability of miRNA. The limitations of these studies have recently been reviewed and include lack of miRNA encapsulation in exosomes, first-passage elimination of some miRNA in intestinal mucosa and liver, sample degradation and biased bioinformatics protocols (Zempleni et al. 2019).

Exosome and RNA-defined diets elicited gene expression changes in murine placentas, which is consistent with the accumulation of dietary BMEs and miRNA cargos in the placenta and depletion of 
milk-enriched miRNA in placentas from mice fed the ERD diet. The expression of mRNAs implicated in extracellular matrix (ECM) components and cell adhesion pathways was more abundant in ERS placentas than in ERD placentas and explains the greater reproductive success, judged by litter size in ERS breeders compared to ERD breeders. The $17 \mathrm{mRNAs}$ that were differentially expressed in ERS and ERD placentas have 35 putative miRNA binding sites, including binding sites for nine miRNA present in BMEs (Benmoussa et al. 2020). Future studies will need to identify the miRNA that alter the expression of ECM components, for example, by knocking out the expression of distinct miRNA. We currently cannot explain why the size of litters born to males fed the ERD diet was smaller than ERS controls. We speculate that dietary depletion of miRNA may reduce sperm quality through epigenetic mechanisms (Shin et al. 2011, Zhang et al. 2019). The preliminary results of this study confirmed the paternal effect of ERD diet on litter size as well, but the main goal of the current research was addressing the maternal effect of ERD on the embryo and placenta growth. We did not formally assess the cause of the increased mortality of pups born to ERD parents. We speculate that changes in maternal care, aberrant prenatal development or low nutritional quality of milk might have caused the observed increase in mortality. These factors are not mutually exclusive. We propose that the lower weight of placentas from ERD dams compared to ERS dams was caused by a decrease in the expression of ECM components in placenta, which is consistent with a previous report implicating ECM proteins in placenta growth (Franczyk et al. 2017, Lv et al. 2018).

While this study suggested a strong detrimental effect of BMEs and micRNA cargo depletion on fecundity in mice, epidemiological evidence is inconclusive. One study reported an at best modest effect of dairy consumption on reproductive success in Denmark and North America (Wise et al. 2017) and another study reported a small positive effect of dairy consumption on fertility in women older than 35 years undergoing in vitro fertilization (Afeiche et al. 2016). These studies did not assess BMEs and their miRNA cargos as independent variables due to a lack of awareness and absence of information on BMEs and their cargos in dairy composition databases. Note that the content of miRNA differs substantially between liquid milk and other dairies (Howard et al. 2015). Collectively, it would be premature to conclude as to whether consumption of dairy or BMEs alters fecundity in human populations.

We used ERD and ERS diets to assess effects of milk BMEs and their cargos on placental gene expression and litter size in mice. In these diets, all ingredients are identical but the number, bioavailability and cargos of BMEs are decreased by ultrasonication (see Materials and methods section). The alternative would have been to remove exosomes by ultracentrifugation or chromatography protocols, but these protocols have the disadvantage of low yield and loss of extracellular vesicles other than exosomes, for example, fat globules, lipoproteins and casein micelles. We have begun documenting the content of exosomes and cargos in ERD and ERS and human milk to maximize transparency and reproducibility (Leiferman et al. 2019, Sukreet et al. 2019). Previous studies suggest that food and water intake, respiratory exchange ratio, physical activity and serum markers of liver and kidney function are not different in mice fed ERD or ERS diets (Leiferman et al. 2018). In this study, the diets had the desired effects and caused an up to $80 \%$ depletion of miRNA levels in placentas fed ERD compared to ERS controls.

In conclusion, this study suggests that dietary BMEs and their miRNA cargos are bioavailable and accumulate in the placenta and embryo in mice. BMEs-dependent changes in gene expression appear to promote embryonic growth and survival. This study is a depletion study and it would be inappropriate to draw the conclusion that supplementation with BMEs beyond of what is a nutritionally normal intake of BMEs will have benefits regarding fecundity. Causal connections between distinct miRNA and fecundity will need to be tested in miRNA knockout mice.

\section{Supplementary materials}

This is linked to the online version of the paper at https://doi. org/10.1530/REP-19-0521.

\section{Declaration of interest}

J Z serves as a consultant for PureTech Health, Inc. The other authors have nothing to disclose.

\section{Funding}

The study was supported by awards from the National Institute of Food and Agriculture (NIFA), United States Department of Agriculture (USDA, 2015-67017-23181 and 2016-6700125301), the National Institutes of Health grant 1P20GM104320, a Food for Health grant by the Nebraska University President's Office, USDA Hatch-1011996, and USDA multistate group W-4002.

\section{Author contribution statement}

$M S$ and $J Z$ designed research. M S performed research. M S, J S, S D K, J C and J Z analyzed data. M S wrote draft versions of the paper. S D K and J C edited the paper. J Z conceived the project and wrote the final version of the paper. 


\section{Acknowledgements}

The authors thank Biomedical and Obesity Research Core (National Institutes of Health, NIH P20GM104320) at the University of Nebraska-Lincoln for assistance with sample analysis. The authors thank the DNA Sequencing Core at the University of Nebraska Medical Center (NIH grants P20GM103427-14 and 1P30GM110768-01, and a grant from the Fred \& Pamela Buffett Cancer Center NIH P30CA036727) for mRNA sequencing analysis. This publication's contents are the sole responsibility of the authors and do not necessarily represent the official views of the NIH. The authors thank David Giraud (Department of Nutrition and Health Sciences) for technical assistance.

\section{References}

Afeiche MC, Chiu YH, Gaskins AJ, Williams PL, Souter I, Wright DL, Hauser R, Chavarro JE \& EARTH Study Team 2016 Dairy intake in relation to in vitro fertilization outcomes among women from a fertility clinic. Human Reproduction 31 563-571. (https://doi.org/10.1093/ humrep/dev344)

Aguilar-Lozano A, Baier SR, Grove R, Shu J, Giraud D, Leiferman A, Mercer KE, Cui J, Badger TM, Adamac J et al. 2018 Concentrations of purine metabolites are elevated in fluids from adults and infants and in livers from mice fed diets depleted of bovine milk exosomes and their RNA cargos. Journal of Nutrition 148 1886-1894. (https://doi. org/10.1093/jn/nxy223)

Altmae S, Martinez-Conejero JA, Esteban FJ, Ruiz-Alonso M, StavreusEvers A, Horcajadas JA \& Salumets A 2013 MicroRNAs miR-30b, miR30d, and miR-494 regulate human endometrial receptivity. Reproductive Sciences 20 308-317. (https://doi.org/10.1177/1933719112453507)

Arai KY \& Nishiyama T 2007 Developmental changes in extracellular matrix messenger RNAs in the mouse placenta during the second half of pregnancy: possible factors involved in the regulation of placental extracellular matrix expression. Biology of Reproduction 77 923-933. (https://doi.org/10.1095/biolreprod.107.061382)

Baier SR, Nguyen C, Xie F, Wood JR \& Zempleni J 2014 MicroRNAs are absorbed in biologically meaningful amounts from nutritionally relevant doses of cow's milk and affect gene expression in peripheral blood mononuclear cells, HEK-293 kidney cell cultures, and mouse livers. Journal of Nutrition 144 1495-1500. (https://doi.org/10.3945/ jn.114.196436)

Benmoussa A, Laugier J, Beauparlant CJ, Lambert M, Droit A \& Provost P 2020 Complexity of the microRNA transcriptome of cow milk and milkderived extracellular vesicles isolated via differential ultracentrifugation. Journal of Dairy Science 103 16-29. (https://doi.org/10.3168/jds.201916880)

Benmoussa A, Lee CH, Laffont B, Savard P, Laugier J, Boilard E, Gilbert C, Fliss I \& Provost P 2016 Commercial dairy cow milk microRNAs resist digestion under simulated gastrointestinal tract conditions. Journal of Nutrition 146 2206-2215. (https://doi.org/10.3945/jn.116.237651)

Bernstein E, Kim SY, Carmell MA, Murchison EP, Alcorn H, Li MZ, Mills AA, Elledge SJ, Anderson KV \& Hannon GJ 2003 Dicer is essential for mouse development. Nature Genetics 35 215-217. (https://doi. org/10.1038/ng1253)

Cai M, He H, Jia X, Chen S, Wang J, Shi Y, Liu B, Xiao W \& Lai S 2018 Genome-wide microRNA profiling of bovine milk-derived exosomes infected with Staphylococcus aureus. Cell Stress and Chaperones 23 663-672. (https://doi.org/10.1007/s12192-018-0876-3)

Chen T, Xie MY, Sun JJ, Ye RS, Cheng X, Sun RP, Wei LM, Li M, Lin DL, Jiang QY et al. 2016 Porcine milk-derived exosomes promote proliferation of intestinal epithelial cells. Scientific Reports 633862. (https://doi.org/10.1038/srep33862)

Franczyk M, Lopucki M, Stachowicz N, Morawska D \& Kankofer M 2017 Extracellular matrix proteins in healthy and retained placentas, comparing hemochorial and synepitheliochorial placentas. Placenta $\mathbf{5 0}$ 19-24. (https://doi.org/10.1016/j.placenta.2016.12.014)
Friedman RC, Farh KK, Burge CB \& Bartel DP 2009 Most mammalian mRNAs are conserved targets of microRNAs. Genome Research 19 92-105. (https://doi.org/10.1101/gr.082701.108)

Golan-Gerstl R, Elbaum Shiff Y, Moshayoff V, Schecter D, Leshkowitz D \& Reif S 2017 Characterization and biological function of milk-derived miRNAs. Molecular Nutrition and Food Research 61 28643865. (https:// doi.org/10.1002/mnfr.201700009)

Gurunathan S, Kang MH, Jeyaraj M, Qasim M \& Kim JH 2019 Review of the isolation, characterization, biological function, and multifarious therapeutic approaches of exosomes. Cells 8 307. (https://doi. org/10.3390/cells8040307)

Howard KM, Jati Kusuma R, Baier SR, Friemel T, Markham L, Vanamala J \& Zempleni J 2015 Loss of miRNAs during processing and storage of cow's (Bos taurus) milk. Journal of Agricultural and Food Chemistry 63 588-592. (https://doi.org/10.1021/jf505526w)

Hromadnikova I, Kotlabova K, Ivankova K \& Krofta L 2017 First trimester screening of circulating C19MC microRNAs and the evaluation of their potential to predict the onset of preeclampsia and IUGR. Plos One 12 e0171756. (https://doi.org/10.1371/journal.pone.0171756)

Huang HY, Lin YC, Li J, Huang KY, Shrestha S, Hong HC, Tang Y, Chen YG, Jin CN, Yu Y et al. 2020 miRTarBase 2020: updates to the experimentally validated microRNA-target interaction database. Nucleic Acids Research 48 D148-D154. (https://doi.org/10.1093/nar/gkz896)

Izumi H, Kosaka N, Shimizu T, Sekine K, Ochiya T \& Takase M 2012 Bovine milk contains microRNA and messenger RNA that are stable under degradative conditions. Journal of Dairy Science 95 4831-4841. (https://doi.org/10.3168/jds.2012-5489)

Kosaka N, Izumi H, Sekine K \& Ochiya T 2010 MicroRNA as a new immune-regulatory agent in breast milk. Silence 1 7. (https://doi. org/10.1186/1758-907X-1-7)

Kozomara A \& Griffiths-Jones S 2014 miRBase: annotating high confidence microRNAs using deep sequencing data. Nucleic Acids Research 42 D68-D73. (https://doi.org/10.1093/nar/gkt1181)

Kusuma Jati RJ, Manca S, Friemel T, Sukreet S, Nguyen C \& Zempleni J 2016 Human vascular endothelial cells transport foreign exosomes from cow's milk by endocytosis. American Journal of Physiology: Cell Physiology 310 C800-C807. (https://doi.org/10.1152/ajpcell.00169.2015)

Leiferman A, Shu J, Grove R, Cui J, Adamec J \& Zempleni J 2018 A diet defined by its content of bovine milk exosomes and their RNA cargos has moderate effects on gene expression, amino acid profiles and grip strength in skeletal muscle in C57BL/6 mice. Journal of Nutritional Biochemistry 59 123-128. (https://doi.org/10.1016/j. jnutbio.2018.06.007)

Leiferman A, Shu J, Upadhyaya B, Cui J \& Zempleni J 2019 Storage of extracellular vesicles in human milk, and microRNA profiles in human milk exosomes and infant formulas. Journal of Pediatric Gastroenterology and Nutrition 69 235-238. (https://doi.org/10.1097/ MPG.0000000000002363)

Liao Y, Du X, Li J \& Lonnerdal B 2017 Human milk exosomes and their microRNAs survive digestion in vitro and are taken up by human intestinal cells. Molecular Nutrition and Food Research 61 11. (https:// doi.org/10.1002/mnfr.201700082)

Lv C, Yu WX, Wang Y, Yi DJ, Zeng MH \& Xiao HM 2018 MiR-21 in extracellular vesicles contributes to the growth of fertilized eggs and embryo development in mice. Bioscience Reports 38 BSR20180036. (https://doi.org/10.1042/BSR20180036)

Machtinger R, Laurent LC \& Baccarelli AA 2016 Extracellular vesicles: roles in gamete maturation, fertilization and embryo implantation. Human Reproduction Update 22 182-193. (https://doi.org/10.1093/ humupd/dmv055)

Manca S, Upadhyaya B, Mutai E, Desaulniers AT, Cederberg RA, White BR \& Zempleni J 2018 Milk exosomes are bioavailable and distinct microRNA cargos have unique tissue distribution patterns. Scientific Reports 8 11321. (https://doi.org/10.1038/s41598-018-29780-1)

Munagala R, Aqil F, Jeyabalan J \& Gupta RC 2016 Bovine milk-derived exosomes for drug delivery. Cancer Letters 371 48-61. (https://doi. org/10.1016/j.canlet.2015.10.020)

Reeves PG 1993 Components of the AIN-93 diets as improvements in the AIN-76A diet. Journal of Nutrition 127 838S-841S. (https://doi. org/10.1093/jn/127.5.838S)

Schanzenbach CI, Kirchner B, Ulbrich SE \& Pfaffl MW 2017 Can milk cell or skim milk miRNAs be used as biomarkers for early pregnancy 
detection in cattle? PLoS ONE 12 e0172220. (https://doi.org/10.1371/ journal.pone.0172220)

Shin JY, Gupta MK, Jung YH, Uhm SJ \& Lee HT 2011 Differential genomic imprinting and expression of imprinted microRNAs in testes-derived male germ-line stem cells in mouse. PLOS ONE 6 e22481. (https://doi. org/10.1371/journal.pone.0022481)

Shu J, Chiang K, Zempleni J \& Cui J 2015 Computational characterization of exogenous microRNAs that can be transferred into human circulation. PLOS ONE 10 e0140587. (https://doi.org/10.1371/journal. pone.0140587)

Sohel MM, Hoelker M, Noferesti SS, Salilew-Wondim D, Tholen E, Looft C, Rings F, Uddin MJ, Spencer TE, Schellander K et al. 2013 Exosomal and non-exosomal transport of extra-cellular microRNAs in follicular fluid: implications for bovine oocyte developmental competence. PLOS ONE 8 e78505. (https://doi.org/10.1371/journal.pone.0078505)

Sukreet S, Silva BVREB, Adamec J, Cui J \& Zempleni J 2019 Sonication and short-term incubation alter the content of bovine milk exosome cargos and exosome bioavailability. Current Developments in Nutrition 3 (Supplement 1) OR26-08-19. (https://doi.org/10.1093/cdn/nzz033.OR26-08-19)

Sun J, Aswath K, Schroeder SG, Lippolis JD, Reinhardt TA \& Sonstegard TS 2015 MicroRNA expression profiles of bovine milk exosomes in response to Staphylococcus aureus infection. BMC Genomics 16806. (https://doi.org/10.1186/s12864-015-2044-9)

Takov K, Yellon DM \& Davidson SM 2017 Confounding factors in vesicle uptake studies using fluorescent lipophilic membrane dyes. Journal of Extracellular Vesicles 6 1388731. (https://doi.org/10.1080/20013078.20 17.1388731)

Vilella F, Moreno-Moya JM, Balaguer N, Grasso A, Herrero M, Martinez S, Marcilla A \& Simon C 2015 Hsa-miR-30d, secreted by the human endometrium, is taken up by the pre-implantation embryo and might modify its transcriptome. Development 142 3210-3221. (https://doi. org/10.1242/dev.124289)

Wang L, Sadri M, Giraud D \& Zempleni J 2018 RNase H2-dependent polymerase chain reaction and elimination of confounders in sample collection, storage, and analysis strengthen evidence that microRNAs in bovine milk are bioavailable in humans. Journal of Nutrition $\mathbf{1 4 8}$ 153-159. (https://doi.org/10.1093/jn/nxx024)

Wise LA, Wesselink AK, Mikkelsen EM, Cueto H, Hahn KA, Rothman KJ, Tucker KL, Sorensen HT \& Hatch EE 2017 Dairy intake and fecundability in 2 preconception cohort studies. American Journal of Clinical Nutrition 105 100-110. (https://doi.org/10.3945/ajcn.116.138404)

Wolf T, Baier SR \& Zempleni J 2015 The intestinal transport of bovine milk exosomes is mediated by endocytosis in human colon carcinoma caco-2 cells and rat small intestinal IEC-6 cells. Journal of Nutrition $\mathbf{1 4 5}$ 2201-2206. (https://doi.org/10.3945/jn.115.218586)

Yanez-Mo M, Siljander PR, Andreu Z, Zavec AB, Borras FE, Buzas EI, Buzas K, Casal E, Cappello F, Carvalho J et al. 2015 Biological properties of extracellular vesicles and their physiological functions. Journal of Extracellular Vesicles 4 27066. (https://doi.org/10.3402/jev.v4.27066)

Zempleni J, Sukreet S, Zhou F, Wu D \& Mutai E 2019 Milk-derived exosomes and metabolic regulation. Annual Review of Animal Biosciences 7 245-262. (https://doi.org/10.1146/annurev-animal-020518-115300)

Zhang JJ, Chandimali N, Kim N, Kang TY, Kim SB, Kim JS, Wang XZ, Kwon T \& Jeong DK 2019 Demethylation and microRNA differential expression regulate plasma-induced improvement of chicken sperm quality. Scientific Reports 9 8865. (https://doi.org/10.1038/s41598-01945087-1)

Zhang L, Hou D, Chen X, Li D, Zhu L, Zhang Y, Li J, Bian Z, Liang X, Cai X et al. 2012 Exogenous plant MIR168a specifically targets mammalian LDLRAP1: evidence of cross-kingdom regulation by microRNA. Cell Research 22 107-126. (https://doi.org/10.1038/cr.2011.158)

Received 1 November 2019

First decision 19 December 2019

Revised manuscript received 16 June 2020

Accepted 2 July 2020 